\title{
An Energy Efficient Protocol To Increase Network Life In WSN
}

\author{
Rishu Gupta ${ }^{1}$, Mrs. Tripti Malhotra ${ }^{2}$ \\ ${ }^{l}$ (ECE Dept, D.I.E.T/ KUK,INDIA) \\ ${ }^{2}$ (ECE Dept, D.I.E.T/ KUK,INDIA)
}

\begin{abstract}
Wireless Sensor Network consists of several sensor nodes, these nodes loss some of their energy after the process of communication. So an energy efficient approach is required to improve the life of the network. In case of broadcast network, LEACH protocol uses an aggregative approach by creating cluster of nodes. Now the major concern is to built such clusters over WSN in an optimized way. This work presents the improvement over LEACH protocol. Hence we have different work environments where the network is having different capacities. The proposed work shows how the life time of the network will improve when the number of nodes varies within the network.
\end{abstract}

Keywords - LEACH, energy, lifetime, cluster based, WSM

\section{INTRODUCTION}

A WSN Consists of tiny sensor nodes used to monitor physical and environmental conditions such as temperature, pressure, sound etc. These are basically used to pass their data through the network to a main location. The development in this field was motivated by military applications such as battlefield surveillance; today such networks are used in many applications such as environment and habitat monitoring, health care applications, home automations, traffic control etc.

It is an sensing technology where tiny, autonomous and compact devices called sensor nodes or motes are deployed in a remote area to detect phenomena, collect and process data and transmit sensed information to users.

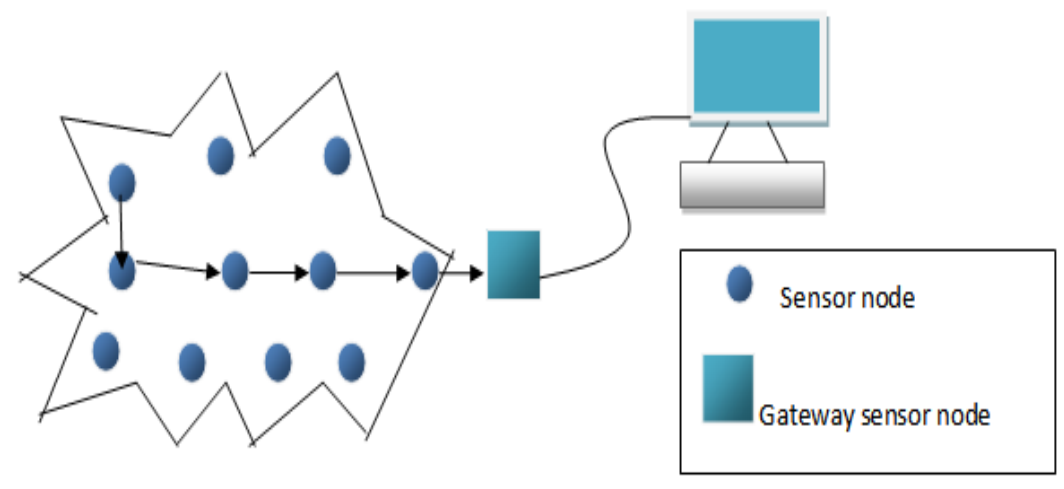

Figure 1. Structure of wsn

Each network sensor node is made up of four basic components-a sensing unit, a processing unit, a transceiver unit and a power unit or some energy source usually a battery.

Size of the sensor motes will vary from that of a shoe box down to the size of a grain of dust. Similarly cost of sensor nodes may range from hundreds of dollars to a few pennies, depending on the complexity of the individual sensor nodes. The cost and size constraints on sensor nodes results in corresponding constraints on various resources such as energy, computational speed, memory and communication bandwidth. The topology of these sensor networks can vary from a simple star to a multihop mesh network. Sensors integrated into the structures and the environment could provide tremendous benefits to society.

The ideal wireless sensor is networked and scalable, consumes very little power, capable of fast data acquisition, accurate and reliable, having less cost and requires no real maintenance. A wireless sensor network generally consists of a base station (or "gateway") that can communicate with a number of wireless sensors via a radio link. Firstly the data is collected at the wireless sensor node, compressed and then transmitted to gateway directly or other sensor nodes may be used to forward data to the gateway. At last it is presented to the system by the gateway. Selection of sensors and communication links requires knowledge of the problem definition and applications. Major design considerations are battery life, sensor update rate and size. 


\subsection{Characteristics of WSN}

The main characteristics of WSN includes-

A. Power consumption constraints for nodes using batteries or energy harvesting

B. Ability to cope with node failures

C. Mobility of nodes

D. Communication failures

E. Heterogeneity of nodes

F. Scalability to large scale of deployment.

G. Ability to withstand harsh environmental conditions

H. Ease of use.

\subsection{Architecture of WSN}

The general architecture for WSN follows the OSI model. Basically in sensor network we need five layers: Physical layer, data link layer, network layer, transport layer, application layer. In addition to these layers there exist also three cross layer planes. This can be seen in figure given.

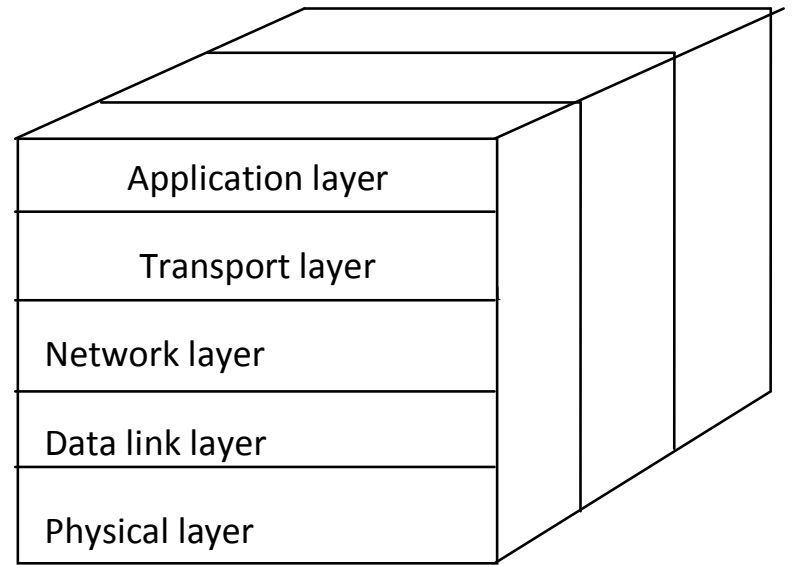

The three cross planes or layers are named as power management plane, mobility management plane and task management plane. These layers are used to manage the network and also make the sensors work together in order to increase the overall efficiency of the network.

The design of layered architecture would normally consist of a base station and sensors scattered in the field. One of the earliest protocols to complete the implementation of the layered architecture is the UNPF (Unified Network Protocol Framework), multihop sensor network architecture.

\subsection{Routing using Packet Switching Technology}

Routing is the process of selecting paths in a network to send traffic of the network. This article is primarily concerned with routing using packet switching technology in electronic data networks. In packet switching network, the routing directs packet forwarding, the transmission of logically addressed packets from their source toward their destination through intermediate nodes, some hardware devices called switches, routers, gateway or bridges. Information message in form of packets forwarded through the routers, having routing tables which maintains a record of the routes to various network destination. Thus for efficient routing, it is very important to construct routing tables in the routers memory.

\section{Proposed Work}

The proposed work is the improvement in LEACH protocol in terms to decide the next node in the cluster. Basically LEACH protocol is used in case of broadcast or multicast network. It gives the concept of load distribution and cluster based routing where the nodes are interconnected in the form of clusters and respectively perform the communication. In data gathering application, data from all the nodes is required to be collected and transmitted to the base station. A simple approach to perform this data gathering task is for each node to transmit its data directly to BS. Since the BS is typically located far away. So the nodes in a network will die very quickly. Therefore an improved approach is to use to reduce the amount of data that must be transmitted to the base station (sink) in order to reduce energy. This work shows by using EEP protocol the life time of the network will improve. 


\section{Results And Discussions}

The life time of the network basically depends on number of alive nodes. If there is less number of alive nodes life of the network is ended. The simulation parameters which are used to increase the life time of the network are:

1. Dead Nodes.

2. Alive nodes.

3. Packet transmission rate.

Here figure shows the output of the modified system, existing leach output is also considered for the purpose of comparison.

\section{1 scenario one}

In this scenario we are taking $\mathrm{n}=50$ i.e no. of nodes in network is 50 .

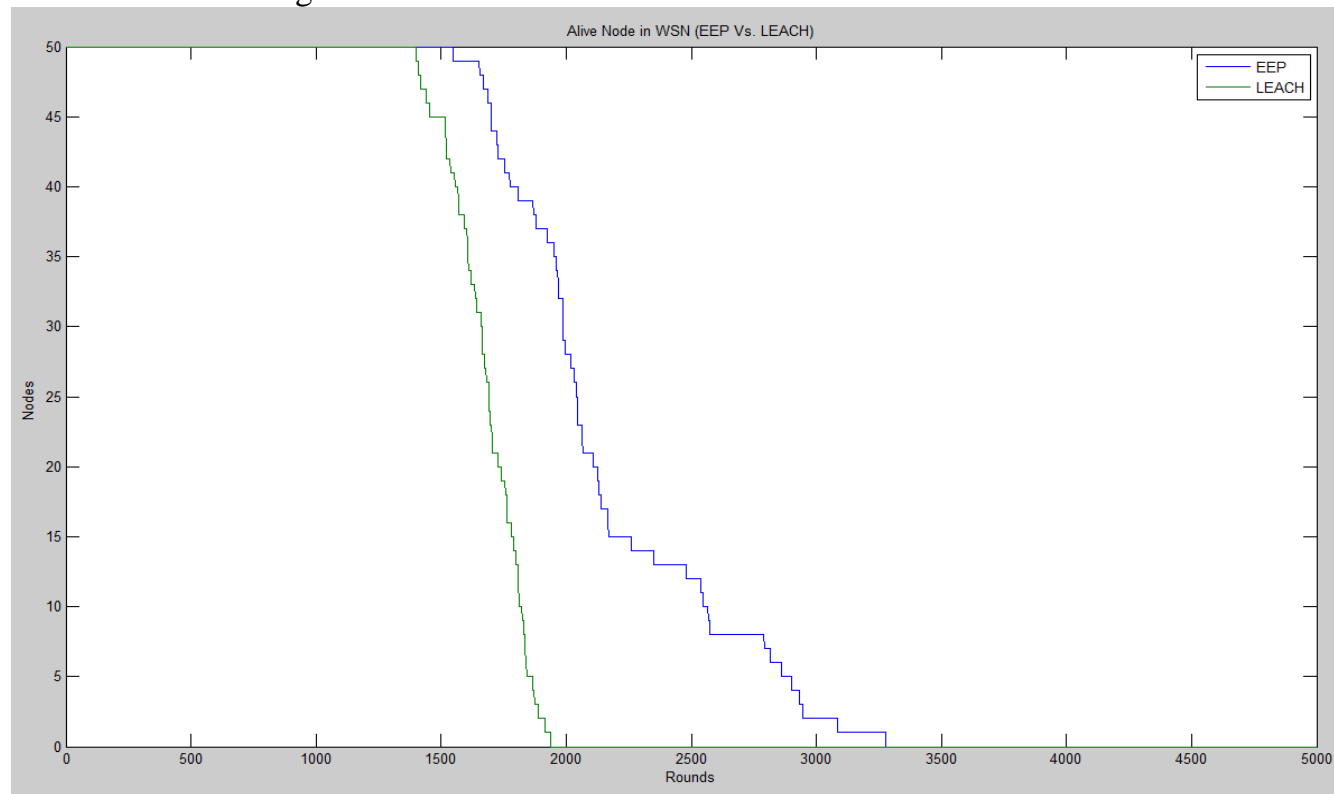

Figure3. Alive node in WSN (EEP Vs LEACH)

The first graph will be of no. of alive nodes in WSN. The life of nodes in EEP is more than the LEACH protocol. In LEACH the nodes are alive between rounds 1400 to 1500 . Up to 1400 rounds the nodes are fully alive in leach. In EEP the nodes are alive between 2400 to 3200 rounds. The next graph will be consists of no. of Dead Nodes in WSN.

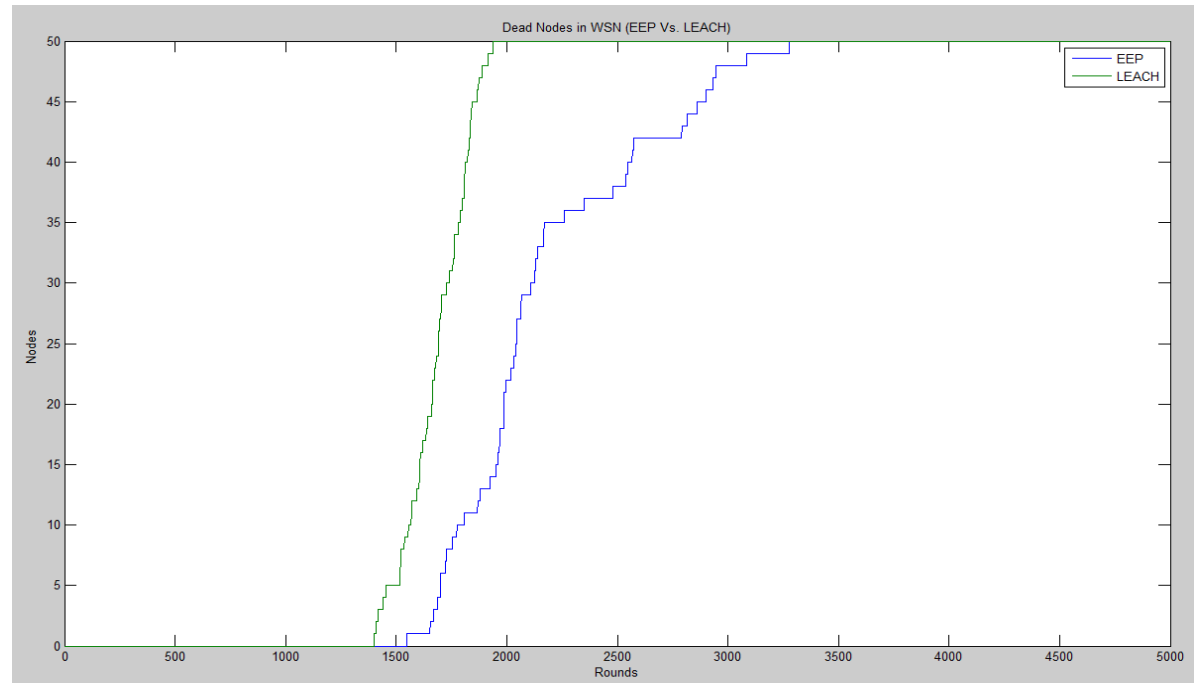

Figure 4. Dead Nodes in WSN(EEP Vs LEACH). 
The life of nodes in LEACH protocol is less than the EEP. In LEACH the nodes starts to die around round 1400 . Up to 1500 rounds the network is fully dead in leach. In EEP the nodes are alive between 2400 to 3200 rounds.

Now we will see the number of packets transmitted to base station in both LEACH and EEP. The number of packets transmitted to base station in EEP is higher than the LEACH protocol. So for a 50 nodes network the efficiency is more given by an EEP protocol than the LEACH protocol

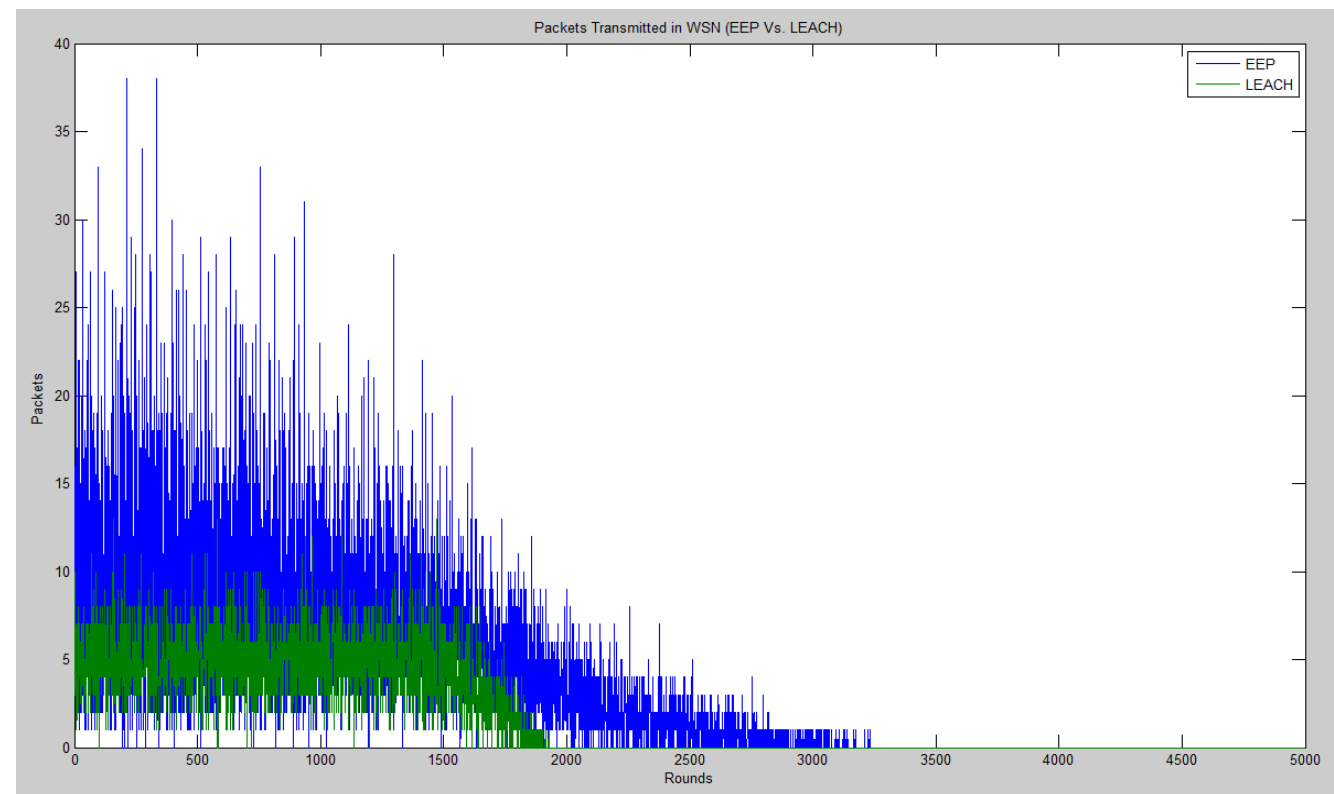

Figure 5. Packet Transmission in WSN (EEP Vs LEACH).

\section{2 scenario two}

In this scenario we are taking $n=100$ i.e no. of nodes in network is 100 . First we will see the number of Alive Nodes in WSN.

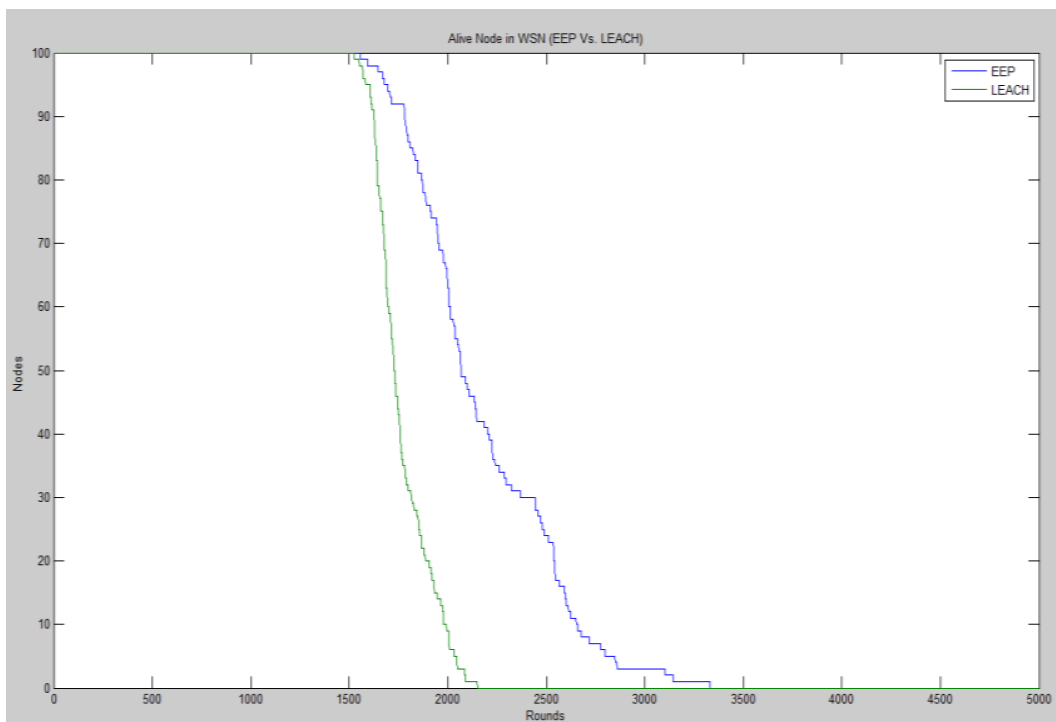

Figure6. Alive node in WSN (EEP Vs LEACH)

The life of nodes in EEP is more than the LEACH protocol. In LEACH the nodes are alive between rounds 1500 to 2100 . Up to 1500 rounds the nodes are fully alive in leach. In EEP the nodes are alive between 2100 to 3600 rounds. However as compared to 50 nodes network the network life of LEACH also improved and in EEP network life also increased. Now we will see no. of Dead Nodes in WSN. 


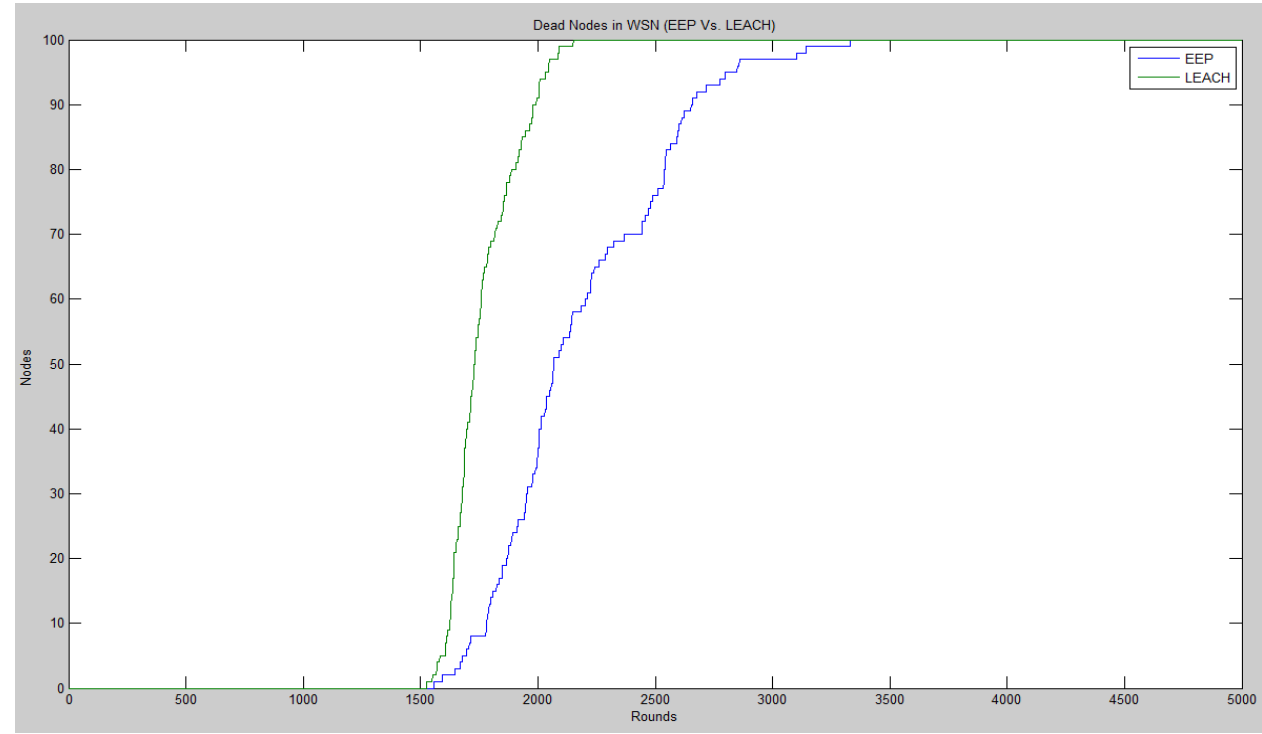

Figure 7. Dead Nodes in WSN(EEP Vs LEACH).

The life of nodes in EEP is more than the LEACH protocol. In LEACH the nodes starts to die around round 1500 . Up to 2100 rounds the network is fully dead in leach. In EEP the nodes are alive between 1600 to 3600 rounds. The network life of 100 nodes network is highly increased in EEP than the LEACH protocol.

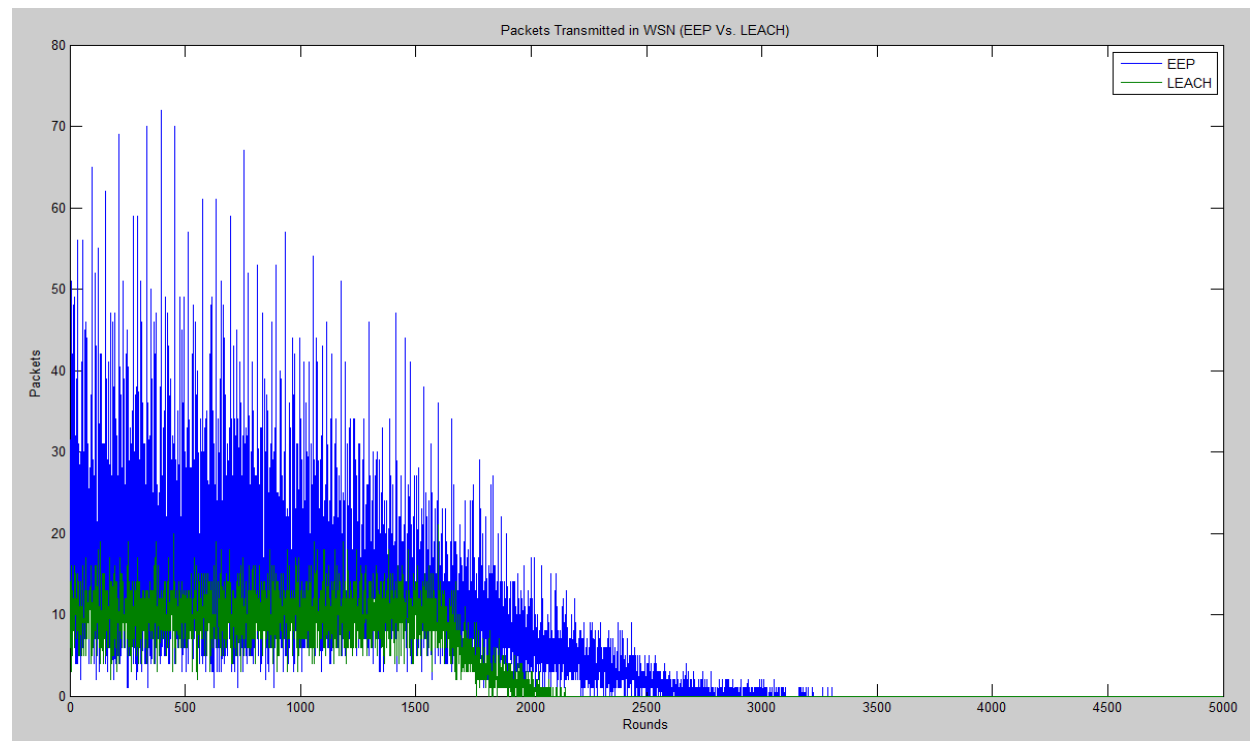

Figure 8. Packet Transmission in WSN (EEP Vs LEACH).

The number of packets transmitted to base station in EEP is higher than the LEACH protocol.

\section{3 scenario three}

In this scenario we are taking $n=150$ i.e no. of nodes in network is 150 . First we will see the number of Alive Nodes in WSN. 


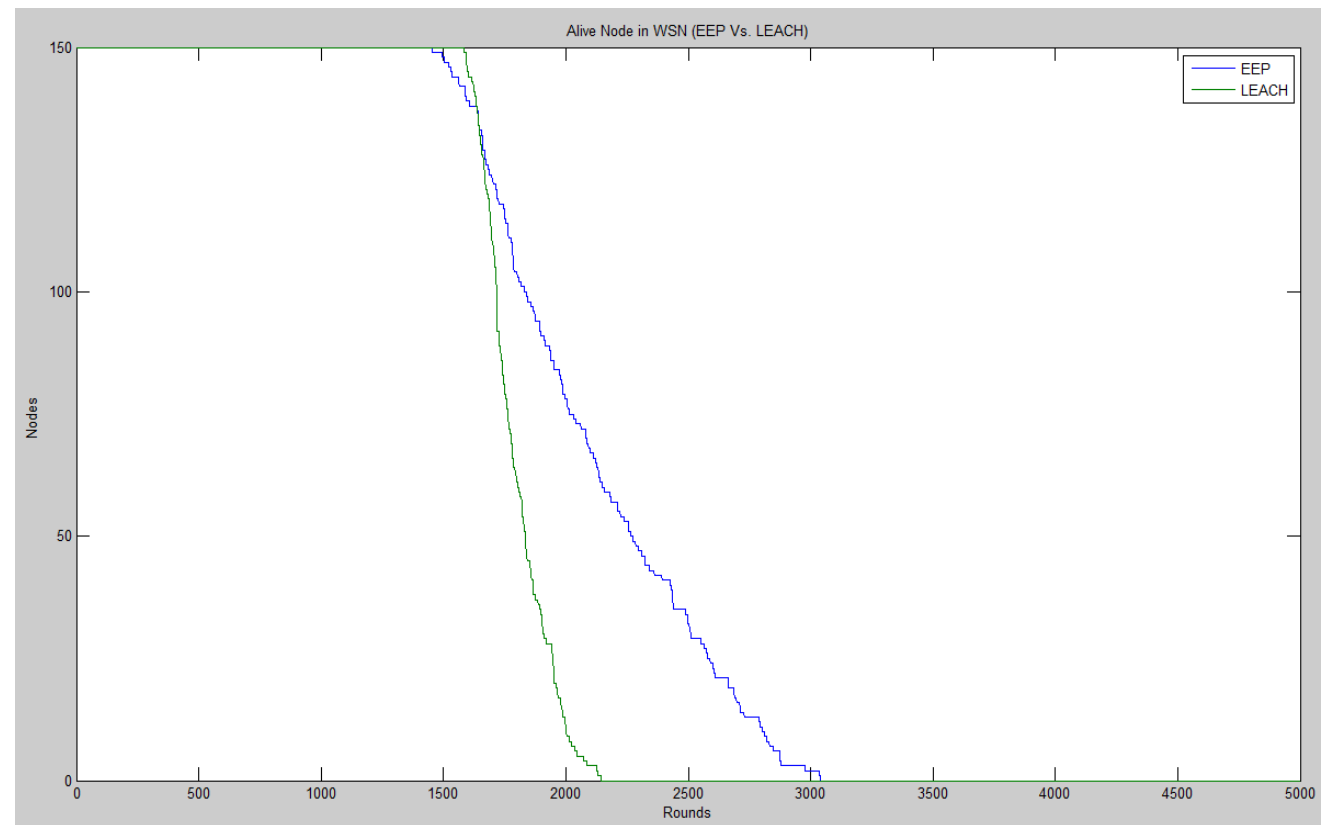

Figure9. Alive node in WSN (EEP Vs LEACH)

In LEACH the nodes are alive between rounds 1500 to 2100 . Up to 1500 rounds the nodes are fully alive in leach. In EEP the nodes are alive between 1450 to 3100 rounds. However as compared to 100 nodes network the network life of EEP network is decreased.

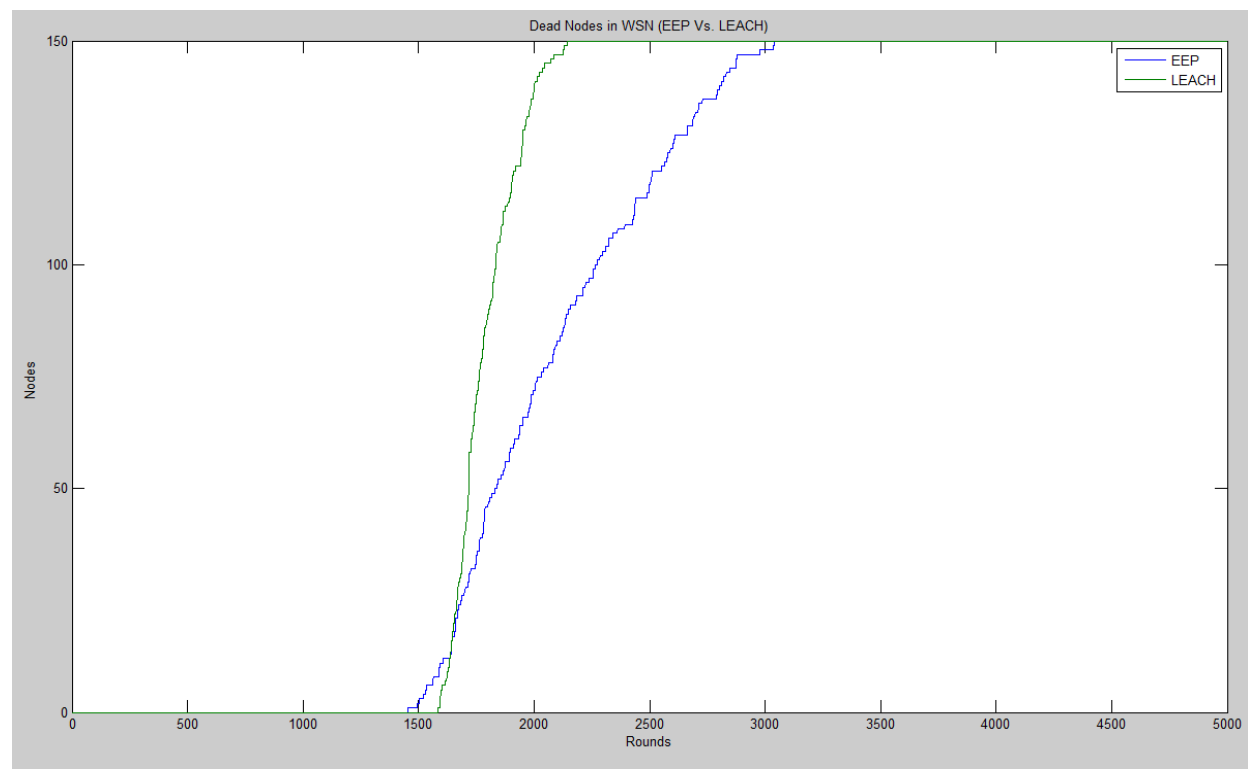

Figure 10 . Dead Nodes in WSN (EEP Vs LEACH).

The second graph will be of no. of dead nodes in WSN. The life of nodes in EEP is more than the LEACH protocol. In LEACH the nodes starts to die around round 1500. Up to 2100 rounds the network is fully dead in leach. In EEP the nodes are alive between 1450 to 3100 rounds. The network life of 150 nodes network is increased in EEP than the LEACH protocol but nodes starts to die earlier as compared to smaller network. 


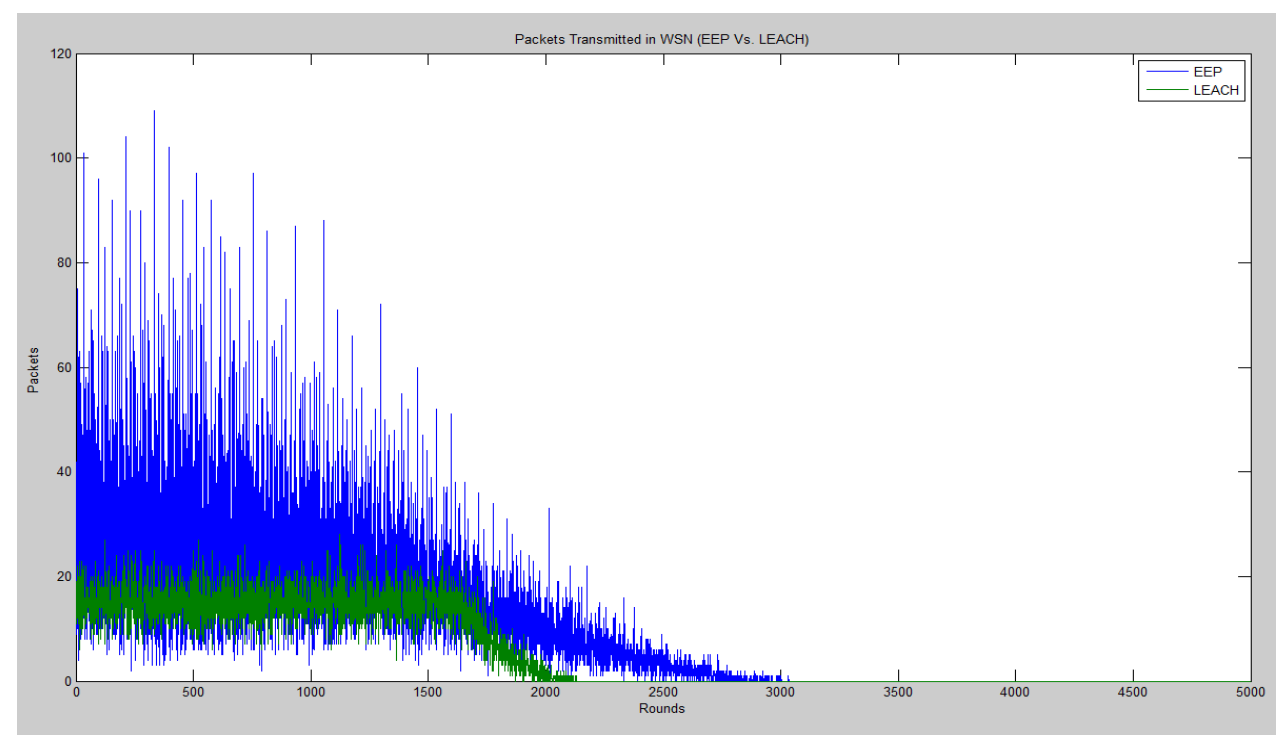

Figure 11. Packet Transmission in WSN (EEP Vs LEACH).

The number of packets transmitted to base station in EEP is higher than the LEACH protocol.

\section{Conclusion}

The main function of a WSN is to gather and send the collected data to a distant BS for further processing and analysis. The result of simulations conducted indicates that the proposed clustering approach is more energy efficient and hence effective in prolonging the network life time compared to LEACH.

\section{Acknowledgements}

The authors would like to thank International organization of scientific research(IOSR-JECE) for supporting this research. The authors would like to thank their colleagues and their family members for many fruitful discussions.

\section{References}

[1] Yingchi Mao, Zhen Liu, Lili Zhang, Xiaofang Li, "An Effective Data Gathering Scheme in Heterogeneous Energy WSNs", International Conference on Computational Science and Engineering,2009.

[2] Kun Zhang and Cong Wang, "A Secure Routing Protocol for Cluster-Based Wireless Sensor Networks Using Group Key Management ", IEEE Xplore,vol.37, pp. 178-185, jan.-2008.

[3] Bolian Yin, Hongchi Shi, and Yi Shang ,"Analysis of Energy Consumption in Clustered Wireless Sensor Networks" IEEE Transactions on Mobile Computing, vol. 3, pp. 272-285, July 2007

[4] Zhao Yulan and Jiang Chunfeng,"Research about Improvement of LEACH Protocol”, An Energy Efficient Protocol for Wireless Sensor Network". Application Research of Computers, No.2 pp: 209-211, Jan. 2005.

[5] Stephan Olariu and Qingwen Xu, "Information Assurance In Wireless Sensor Networks", IEEE Transactions on Mobile Computing, 3(4), pp. 317-331, Dec.2004.

[6] Zenon Chaczko, Ryszard Klempous and Jan Nikodem," Methods of Sensors Localization in Wireless Sensor Network", IEEE Transactions On Mobile Computing, VOL. 3, pp. 3, Jul-Sep 2004.

[7] Adrian Perrig, John Stankovic and David Wagner," Security in wireless sensor network”,. Vol. 47, pp.6-9, June 2004.

[8] Jan Steffan ,Ludger Fiege, Mariano Cilia and Alejandro Buchmann, "Scoping in Wireless Sensor Networks", IEEE Network, 18(1), pp.15-21, May 2004.

[9] Shao-Long and Xing Tao, "Cluster-based power efficient time synchronization in wireless sensor networks", IEEE INFOCOM Conf, Mar. 2004 .

[10] Peng-Jun Wan, Member and Chih-Wei Yi, “Coverage by Randomly Deployed Wireless Sensor Networks", IEEE Wireless Communications and Networking Conference, pp.16-20,Mar. 2003. 